\section{耍 Heighten Science \\ P U B L I C I T I O N S Corporation ISSN 2576-9537}

\title{
Laparoscopic Cholecystectomy: Challenges faced by beginners our perspective
}

\author{
Kunal Chowdhary, Gurinder Kaur, Kapil Sindhu, Muzzafar \\ Zaman*, Aliya Shah, Rohit Dang, Ashish Kumar, Jose John \\ Maiakal and Ashutosh Bawa \\ Doctor, Maharishi Markandeshwar Institute of Medical Sciences and Research, Mullana, \\ Ambala, Haryana, India
}

*Address for Correspondence: Muzzafar Zaman, Doctor, Maharishi Markandeshwar Institute of Medical Sciences and Research, Mullana, Ambala, Haryana, India, Tel: +91-8059931554; Email: muzzafarzaman@yahoo.com

Submitted: 02 August 2018

Approved: 22 August 2018

Published: 23 August 2018

Copyright: @ 2018 Chowdhary K, et al. This is an open access article distributed under the Creative Commons Attribution License, which permits unrestricted use, distribution, and reproduction in any medium, provided the original work is properly cited

Keywords: Laparoscopic Cholecystectomy; Beginners; Technical Challenges

\section{Abstract}

Background: Laparoscopic cholecystectomy is gold standard and most widely performed surgery for gallstone disease all over the world. Surgeons entering into the field of laparoscopic surgery for the first time faces challenges that are different from those with experienced hands. We in this study tried to enumerate the various such challenges and also recommend few steps to counter them.

Aims \& Objectives: To study the challenges faced by new surgeons in laparoscopic cholecystectomy and recommendations to reduce them.

Material \& Methods: This study was carried out in a medical college in the department of General and Minimal Access surgery. In this retrospective study, ten general surgeons working as senior residents in in this medical college over a period of 3 years having never performed laparoscopic surgery in past were included.

Results: A total of 50 cases, five operated by each surgeon with minimal assistance by senior surgeon in few cases. Operative time varied from 90 to 120 minutes. The various technical challenges faced by the new surgeon were in the Creation of Pneumoperitoneum, Creation of second port (epigastric port $10 \mathrm{~mm}$ ), Gallbladder Retraction and Dissection at calot's triangle, Dissection at gallbladder bed and Removal of the gallbladder from epigastric port.it has been observed that following various simple steps will abate these technical difficulties for these beginners while doing laparoscopic cholecystectomy.

Conclusion: Laparoscopic cholecystectomy is the most commonly performed minimal access surgical procedure nowadays and almost all the new surgeons enter the world of laparoscopic surgery via this surgery. Knowing and following the above recommendations will help them abate the technical challenges generally faced during the initial phase in the laparoscopic field.

\section{Introduction}

Laparoscopic cholecystectomy has become the gold standard treatment for gallstone disease all over the world. It is the most widely performed surgery by the general surgeons and also the first laparoscopic surgery performed by a new surgeon. Though it may be a routine surgery for an experienced laparoscopic surgeon but for a surgeon entering into the field of laparoscopic surgery for the first time challenges are different. We in this study try to enumerate the various such challenges and also advise few steps to counter them.

\section{Aims and objectives}

To study the challenges faced by new surgeons in laparoscopic cholecystectomy and recommendations to reduce them. 


\section{Material and Methods}

This is a retrospective study in which ten general surgeons working as senior resident in a rural medical college over a period of 3 years having not done laparoscopic surgery in past were included.

\section{Inclusion criteria}

A total of 50 gallstone disease cases in which laparoscopic cholecystectomy was successfully done by new registrars in general surgery were included.

\section{Exclusion criteria}

All other laparoscopic surgeries, those which were converted to open were excluded.

\section{Procedure}

After routine workup all cases were subjected to standard 4 port laparoscopic cholecystectomy under the supervision of senior surgeon. Pneumoperitoneum was created using veress needle via a small cut sufficient for veress needle insertion infra/supra umbilical location depending on surgeons' reference. Post pneumoperitoneum creation the same incision extended to around $11 \mathrm{~mm}$ for entry of the camera trocar. Telescope inserted first visualizing the peritoneal cavity for any injury caused by veress needle. Next epigastric port $10 \mathrm{~mm}$ made first by giving a transverse incision at a point in midline that is corresponding to the inferior edge of the liver and then using sharp trocar entry under vision just to the right of falciform ligament. Other two $5 \mathrm{~mm}$ ports created subcostally one in midclavicular line and other in anterior axillary line. Gall bladder grasped with $4^{\text {th }}$ port and retracted towards right shoulder of the patient to visualize the gallbladder neck and calot's triangle. Grasping the infundibulum of gallbladder with $3^{\text {rd }}$ port and retracting it downwards and laterally dissection at calot's triangle started first posteriorly than anteriorly to free all the fibrofatty tissue in hepatocystic triangle visualizing the cystic duct and artery. Both cystic duct and artery were ligated using clips or sutures and then cut. Gallbladder dissected of the liver bed using monopolar cautery hook. Haemostasis achieved at liver bed and gallbladder taken out from epigastric port in doing so sometimes epigastric port was enlarged that was later on closed using vicryl. After removal of gallbladder final inspection of gallbladder fossa done and abdominal drain no. 20 kept there and taken out from $4^{\text {th }}$ port. All incision closed and drain fixed. Bandage done.

Post-surgery, surgeons' experiences in form verbal questionnaire were noted and used for the study.

\section{Results}

A total of 50 cases, five operated by each surgeon with minimal assistance by senior surgeon in few cases. Operative time varied from 90 to 120 minutes. Few problems faced by all surgeons are enumerated:

\section{Creation of pneumoperitoneum}

All surgeons faced difficulty in creation of pneumoperitoneum via veress needle due to the following reasons:

1. Inability to make a firm grip to lift the abdominal wall of the underlying viscera before insertion of veress needle.

2. Unfamiliarity with the feel of loss of resistance as the veress needle enters the peritoneal cavity.

In spite of various test being performed to confirm the correct placement of verees needle mentioned in books no surgeon was sure that he's in the right place or he has 
not injured any viscera while entering till the time pneumoperitoneum is actually achieved and he has inspected the location by putting the scope. Maximum operative time was wasted in this exercise.

\section{Creation of second port (epigastric port $10 \mathrm{~mm}$ )}

In few cases it was observed that the epigastric port was created either too low or too high causing difficulty in dissection and clipping of cystic duct and artery. Thought the skin incision was corresponding to the inferior edge of liver but on the peritoneal side trocar position was either too high or low. It was also observed that with a transverse incision and port placement port mobility is restricted adding to the problem.

\section{Gallbladder retraction}

It was observed that in some cases with thick wall or grossly distended gallbladder fundus retraction was difficult because of inability to grip the gallbladder. Even after aspiration of contents with aspiration needle gallbladder fundus kept on slipping causing inconvenience to the surgeon.

\section{Dissection at calot's triangle}

It was observed that starting dissection at calot's triangle first posteriorly and then anteriorly to visualize the critical view of safety was not such simple task for a beginner. Many a times due to the aberrant anatomy the same may lead to bleeding obscuring the view and in many cases the junction of cystic duct with common hepatic duct was not clear causing difficulty in safe dissection needing help of senior surgeons.

\section{Dissection at gallbladder bed}

In the initial few cases all surgeon had difficulty dissecting gallbladder of the liver bed in the right plane resulting in liver bleeds requiring cauterization. In some cases gallbladder opened with spillage of bile and stones adding to the difficulty. One more observation that almost every surgeon mentioned that by the time they were dissecting the liver bed their hand got fatigued and there was a sense of hurrying through the remaining case that led to unnecessary liver bleeds or bile, stone spillage.

\section{Removal of the Gallbladder from epigastric port}

In all the cases gallbladder was removed from the epigastric port and during removal in many cases there was rupture of gallbladder with soiling of the wound with bile and stones causing postoperative surgical site infection of epigastric port. In our study out of 50 cases 23 cases had wound soiling with bile out of which 7 epigastric port got infected? In many cases epigastric port had to be enlarged to deliver the gallbladder needing the port to be closed with vicryl to avoid incisional hernia.

\section{Discussion}

Over 750,000 cholecystectomy are performed each year in the United States.[1,2] Patients benefit from reduced pain, faster return to normal activities, and reduced risk of surgical site infection with a laparoscopic approach compared to an open operation. [3] Bile duct injury rates have increased since the introduction of laparoscopic cholecystectomy, occurring in about 3 per 1,000 procedures performed.[4] Bile duct injuries after cholecystectomy can be life altering complications leading to significant morbidity and cost $[5,6]$.

Author's recommendations to the above citied problems based on review of literature and personnel experience:

\section{Entry technique [7]}

We recommend an open technique for first port placement as this technique is reliable, convenient, fast, easy to learn and reproduce, associated with less 
complications like visceral, vascular injuries. Supraumblical skin grasped with ellis forceps and lifted (Figure 1). Smiling infraumblical incision given and subcutaneous tissue dissected. Umbilical tube grasped with kocher's forceps and dissected till the base where anterior sheath is expose and incised (Figure 2-4) artery forceps used to expand the cut in sheath and open the peritoneum. Keeping the upward traction over the kochers (Figure 4) 10mm trocar inserted under vision inside the peritoneal cavity. A suture may be passed over skin margins around the trocar to prevent air leak.

\section{Epigastric port creation [8]}

Our recommendation for epigastric port creation is using a vertical incision instead of transverse incision giving greater port mobility. The epigastric port should be at the level of inferior edge of the liver towards the left of falciform ligament for proper manipulation at the calots triangle (Figures 1,2).

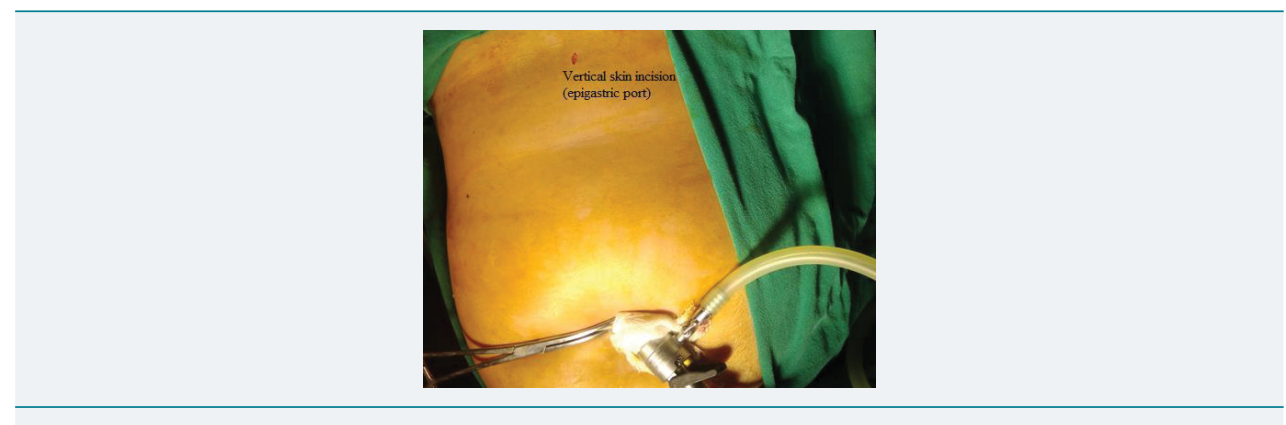

Figure 1: Vertical epigastric port skin incision.

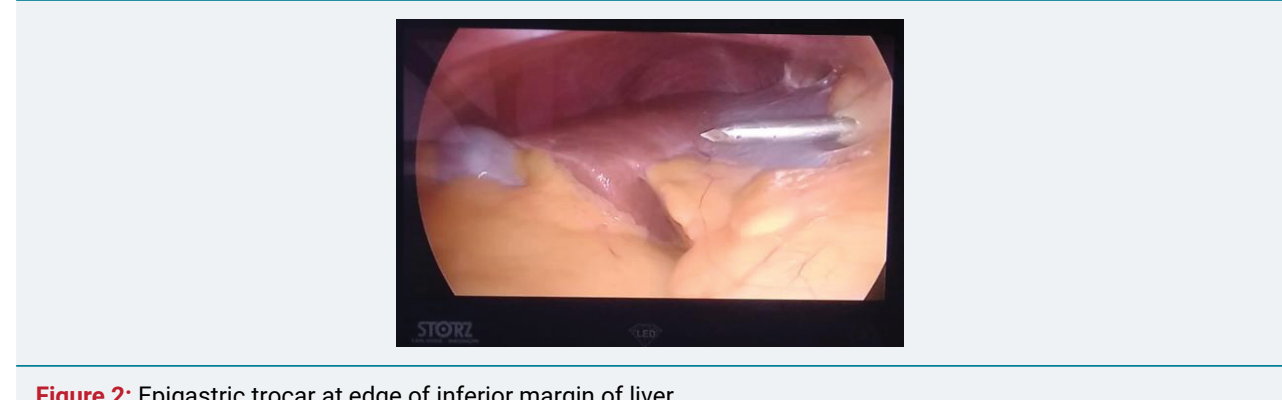

Figure 2: Epigastric trocar at edge of inferior margin of liver.

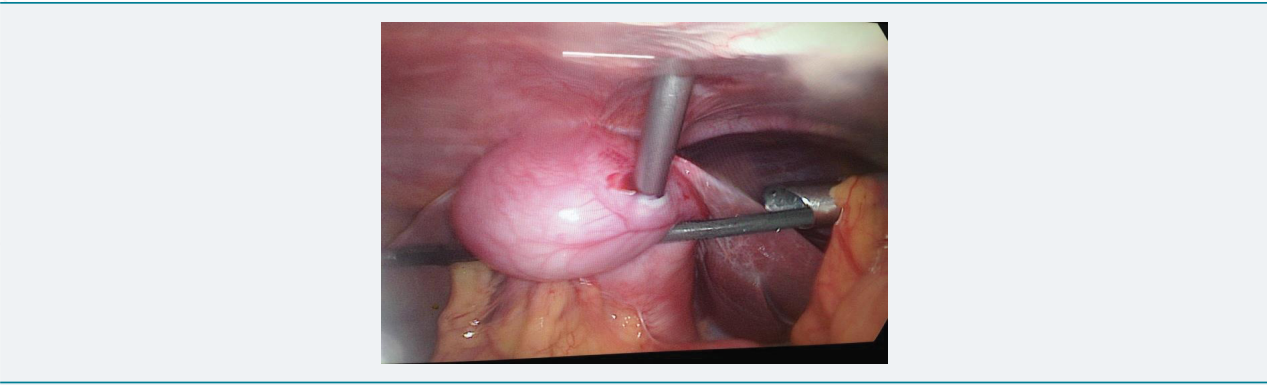

Figure 3: 5mm Trocar inside gallbladder.

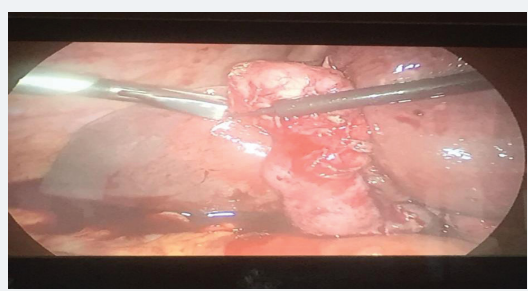

Figure 4: Grasping the gallbladder fundus with toothed grasper. 


\section{Gallbladder retraction [9]}

In cases grossly distended gallbladder fundus retraction can be achieved using aspiration needle to empty the contents facilitating easy grasping of gallbladder. In case where aspiration needle is not available the same can be achieved via pushing the $3^{\text {rd }} 5 \mathrm{~mm}$ cannula with trocar through the fundus of gallbladder under vision and once the port is inside the gallbladder lumen a suction catheter may be inserted into the port after removing the trocar and aspirating the contents. In cases of thick gallbladder wall a toothed grasper may be used for holding the gallbladder fundus or in cases where the above method fails the $4^{\text {th }}$ port may be enlarged to $10 \mathrm{~mm}$ and gallbladder extractor forceps may be used to hold the fundus (Figures 3,4).

\section{Dissection at calot's triangle $[10,11]$}

Dissection at calot's triangle started by first grasping the Hartmann's pouch with a soft grasper lifting it upwards and medially exposing the posterior surface of the gallbladder cystic duct junction. Peritoneum overlying this area is incised and further fatty tissue in this area cleared. Hartmann's pouch than is retracted downwards and laterally and peritoneum covering the anterior surface of gallbladder cystic duct junction incised going upwards. Once the peritoneum is incised and the fatty tissue in both anterior and posterior planes dissected cystic duct and artery can be visualized. Dissection should be at the level of gallbladder neck-cystic duct junction and not at cystic duct-common hepatic duct junction to minimize injuries to the common bile duct. Aim of the dissection at the calots triangle should be to achieve critical view of safety before proceeding with clipping of duct and artery but in cases of aberrant anatomy or frozen calots triangle a fundus first approach may be helpful. If critical view cannot be achieve by any of the above methods a subtotal cholecystectomy is a safer option with similar post-operative outcome rate (Figure 5).

\section{Dissection at gallbladder bed [12]}

The dissection at the gallbladder bed should be in the loose adventitial plane causing minimal damage to the liver and also safeguarding gallbladder from opening and spillage of contents. The right plane can be entered by using adequate traction and counter traction. Also an innovation to decrease the arm fatique of the surgeon is to use an alternate port placement for laparoscopic cholecystectomy. In this the first port remains the same, $2^{\text {nd }}$ port $10 \mathrm{~mm}$ is at mid clavicular line left subcostal margin, $3^{\text {rd }}$ port $5 \mathrm{~mm}$ at epigastric region an $4^{\text {th }}$ port $5 \mathrm{~mm}$ at mid clavicular line right subcostal margin. This trocar placement is ergonomically better with $2^{\text {nd }}, 3$ rd port being the working ports an $4^{\text {th }}$ used for gallbladder fundus retraction (Figure 6).

\section{Removal of the gallbladder from the peritoneal cavity [13]}

Our recommendation is the removal of gallbladder should be in an endobag through the umbilical port. Endobag will prevent the spillage of gallbladder contents into the wound avoiding port site infections and sinus formations. As the umbilical port is made with open access it is easier to pull the gallbladder through this port because of the larger diameter as compared to other ports. After placing the gallbladder in the

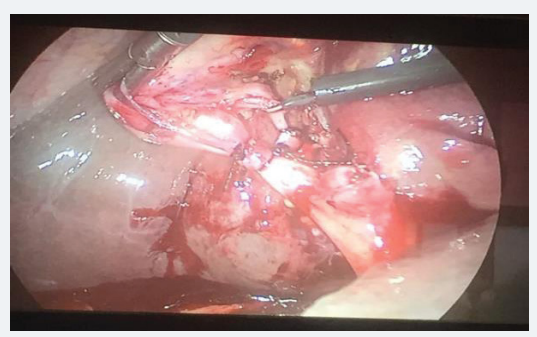

Figure 5: Critical View of safety defined. 


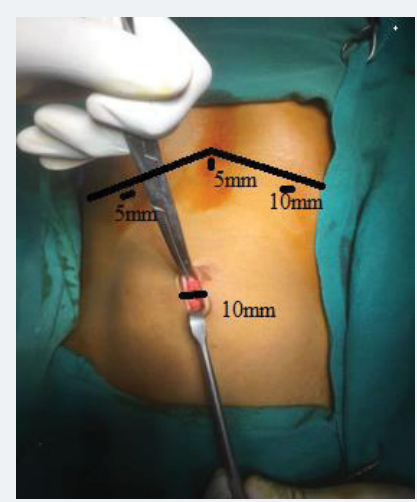

Figure 6: Alternate port placement for laparoscopic cholecystectomy.

endobag the telescope is inserted from the epigastric port and gallbladder extractor entered from the umbilical port taken to subhepatic region endobag grasped pulled through the umbilical port. After removal of gallbladder the umbilical port sheath closure done.

Strasberg first suggested a new surgical strategy called the Critical view safety, to minimize the risk of bile duct injuries in Laparoscopic cholecystectomy. This technique has three main sections:

1. Dissection of the Calot's triangle, including hepatoduodenal ligament,

2. Mobilization of the lowest part of the gallbladder

3. Isolation and identification of the two main structures (cystic duct and cystic artery). In fact, the critical view safety technique is a simple procedure, which can be easily applied by all surgeons cholecystectomy. This technique was implemented by us in all the cases of laparoscopic cholecystectomies.

\section{Conclusion}

Laparoscopic cholecystectomy is the most commonly performed minimal access surgical procedure nowadays and almost all the new surgeons enter the world of laparoscopic surgery via this surgery. Knowing and following the above recommendations will help them abate the technical challenges generally faced during the initial phase in the laparoscopic field.

\section{References}

1. Hurley V, Brownlee S. Cholecystectomy in California: A Close-Up of Geographic Variation. California Healthcare Foundation. 2011.

2. Mac Fadyen $B V$, Jr Vecchio R, Ricardo $A E$, Mathis $C R$. Bile duct injury after laparoscopic cholecystectomy - The United States experience. Surgical Endoscopy. 1998; 12: 315-321. Ref.: https://tinyurl.com/ycwrjxqn

3. Keus F, de Jong JAF, Gooszen HG, van Laarhoven CJHM. Laparoscopic versus open Cholecystectomy for patients with symptomatic cholecystolithiasis. Cochrane Database of Systematic Reviews 2006. Ref.: https://tinyurl.com/y9xhxarc

4. Buddingh KT, Weersma RK, Savenije RA, van Dam GM, Nieuwenhuijs VB. Lower rate of major bile duct injury and increased intraoperative management of common bile duct stones after implementation of routine intraoperative cholangiography. J Am Coll Surg 2011; 213: 267-274. Ref.: https://tinyurl.com/y94qy84n

5. Kern KA. Malpractice litigation involving laparoscopic cholecystectomy. Cost, cause, and consequences. Arch Surg. 1997; 132: 392-397. Ref.: https://tinyurl.com/yapdvljk

6. Flum DR, Flowers $C$, Veenstra DL. A cost-effectiveness analysis of intraoperative Cholangiography in the prevention of bile duct injury during laparoscopic cholecystectomy. J Am Coll Surg. 2003; 196 : 385-393. Ref.: https://tinyurl.com/yasdecak 
7. Lal P, Singh L, Agarwal PN, Kant R. Open Port Placement of the First Laparoscopic Port: A Safe Technique. JSLS. 2004; 8: 364-366. Ref.: https://tinyurl.com/y8n55af9

8. Haribhakti SP, Mistry JH. Techniques of laparoscopic cholecystectomy: Nomenclature and selection. J Minim Access Surg. 2015; 11: 113-118. Ref.: https://tinyurl.com/y7u98gcp

9. Abdullah A Albarrak, Sami Khairy, Alzahrani Mohammed Ahmed. Laparoscopic Cholecystectomy for Acute Calcular Cholecystitis in a Patient with Ventriculoperitoneal Shunt: A Case Report and Literature Review. Case Rep Surg. 2015. Ref.: https://tinyurl.com/y8yjk6xl

10. Vettoretto N, Saronni C, Harbi A, Balestra L, Taglietti L, et al. Critical View of Safety During Laparoscopic Cholecystectomy. JSLS. 2011; 15: 322-325. Ref.: https://tinyurl.com/ybkmpkmj

11. Sorrentino L, Serra F, Cabry F, Gelmini R. Dome down Laparoscopic Cholecystectomy: Our Experience and the State of Art. Annals of Emergency Surgery. 2017; 2: 1022. Ref.: https://tinyurl.com/ybw5e5ua

12. Honda G, Iwanaga T, Kurata M. Dissection of the gallbladder from the liver bed during laparoscopic cholecystectomy for acute or subacute cholecystitis. J Hepatobiliary Pancreat Surg. 2008; 15: 293296. Ref.: https://tinyurl.com/y7ruppyo

13. Majid MH, Meshkat B, Kohar H, El Masry S. Specimen retrieval during elective laparoscopic cholecystectomy: is it safe not to use a retrieval bag? BMC Surgery. 2016; 16: 64. Ref.: https://tinyurl.com/yatrxnyh 